\title{
Synthesis of Paclitaxel Loaded on Nanoparticles of Archaeosomes and Investigation of Its Anti-cancer Properties
}

\author{
Maral Mazloumi Tabrizi ${ }^{1}$, Sepideh Jafarzadeh Rastin², Nahid Kabiri ${ }^{3}$, Azim \\ Akbarzadeh Khiyavi ${ }^{4}$
}

'Department of Toxicology and Pharmacology, Faculty of Pharmacy, Pharmaceutical Sciences Branch, Islamic Azad University, Tehran, Iran. ${ }^{2}$ Department of Medical Genetics, International Campus, Shahid Sadoughi University of Medical Sciences, Yazd, Iran. ${ }^{3}$ Department of Physiology, Faculty of Veterinary Medicine, Shahid Chamran University, Ahvaz, Iran. ${ }^{4}$ Institute Pasteur of Iran, Department of Nanobiotechnology, Tehran, Iran.

\begin{abstract}
Objective: In this study, the effect of archaeosomal paclitaxel drug on breast cancer MDA-MB231 cell lines was evaluated. Material and method: The experiments were conducted to assess the cytotoxicity of paclitaxel in free form and compare it with archaeosomal form of the drug in vitro. To check the size of archaeosomes, Zetasizer device (DLS) was used. The average size of archaeosom nanoparticles containing paclitaxel drugs $430 \mathrm{~nm}$ and surface charge -15 and the average size of control archaeosom nanoparticles without drug $460 \mathrm{~nm}$ and surface charge -14 were reported. The effect of archaeosomal drug containing paclitaxel and free drug were examined by MTT test. Results: In both formulations, zeta potential was negative. Nano-archaeosom containing paclitaxel drug had higher physical stability compared with control nano-Archaeosom. Tests showed the killing effect of nano-archaeosome containing paclitaxel formulation tumor cells was more than free formulation. In the other word, nano-archaeosome containing paclitaxel is more effective than free paclitaxel. Conclusion: The experiments showed that the killing effect of tumor cells of nano-archaeosomes formulations containing paclitaxel was more than free formulation. That is, nano-archaeosomes containing paclitaxel was more effective than free form paclitaxel.
\end{abstract}

Keywords: Nanoparticles- breast cancer-Archaeosome- Paclitaxel- MDA-MB231 cell lines

Asian Pac J Cancer Biol, 2 (3), 67-70

\section{Introduction}

The newest trend in cancer treatment is cancer nanotechnology, the technology helps pharmacists to produce pharmaceutical product with the minimal side effects and the highest therapeutic value. Some of these methods are systematic transfer systems, nanoparticle drugs, intracellular transport, disabled targeting, enabled targeting, and exposure to cell organelles [1]. During the past few decades, research on the drug-carrying particles or small phospholipid vesicles, which is also known as liposomes, has been started. Research demonstrated that nanoliposome carrier can increase the efficacy of the drug at a specified time and reduce toxic and side effects induced by the drug. In 2007, the delivery engineering
Submission Date: 05/13/2017 Acceptance Date: 08/25/2017

was introduced at the University of Louisiana. Through engineering systems, the amount of drug delivery to the tumor at any time can be predicted. By obtaining the time course of drug effects on cancerous tumor, using too much of the drug can be prevented [2]. Considering breast cancer, Jan Park in San Francisco research institute (2005) began to do experiments on drug delivery systems based on nanoliposomes. He noted the purpose of drug delivery systems as increase of drug effectiveness and reduction of the toxicity of anticancer reagents and drugs. Long cycle of carrier macromolecules and nanomolecules like liposomes can enhance the permeability and increase anti-cancer effect. In the past 15 years, paclitaxel has been introduced as one of the best anti-cancer drugs and recently it is known as the best anti-cancer products of

Corresponding Author:

Dr. Azim Akbarzadeh Khyavi and Maral Mazloumi Tabrizi

Institute Pasteur of Iran, Department of Nanobiotechnology, Tehran, Iran.

Department of Toxicology and Pharmacology, Faculty of Pharmacy, Pharmaceutical Sciences Branch, Islamic Azad University, Tehran, Iran.

Email: azimakbarzadeh@pasteur.ac.ir, Ma.mazlumi@gmail.com 
natural origin. Taxanes, with an increase in the mitotic phase, causes cell death and lack of cell proliferation [3]. Due to extremely poor aqueous solubility, paclitaxel was formulated with the carrier, including ethanol and castor oil (cremophor EL) [4]. By increasing the solubility of paclitaxel complex with cyclo dextrin $[5,6]$ combination with Micellar formulation [7], the use of aqueous Astrhlal 2-polyethylene glycol [8] has been attempted. In this study, the effect of archaeosomal paclitaxel drug on breast cancer cell line MDA-MB231 was evaluated.

\section{Materials and Methods}

\section{Extracting the collection of polar lipids}

Liquid medium was used to grow archaea. After growth of Halobacterium salinarum, contents of the flask were centrifuged (speed rpm 6000, for Min 20). Sediment inside the falcon was then weighted by the method of Bligh and Dyer and polar lipids were extracted [77-78-79]. In the first stage, $420 \mathrm{ml}$ water was added per $30 \mathrm{~g}$ of the dry weight of sediment bacteria. In order to complete the disintegration of bacteria, the container was placed on the stirrer for a few hours. Then, the solvent was added,including chloroform, methanol, and water at a ratio of 1: 2: 1. By adding chloroform, total lipid was extracted

\section{Paclitaxel loading on nano-archaeosom}

Cholesterol was added to the solution to make nanoarchaeosom the extracted polar lipids. Since paclitaxel is lipophilicity, it was resolved in solvents such as chloroform or methanol. Then, the solution was placed at room temperature and it was stirrer at speed of rpm 150. Chloroform/methanol solution was re-evaporated.

\section{Paclitaxel loading on pegylated archaeosomes}

This step was the same as previous step but cholesterol and polyethylene glycol (PEG) were added to the mix. Paclitaxel to cholesterol and polyethylene glycol molar ratio were 1,8 and $1 \mathrm{mM}$, respectively.

\section{Making nano-archaeosom without paclitaxel}

All the previous steps were repeated without the presence of paclitaxel and nano-archaeosom was made. Then, cell culture process was performed.

\section{Freezing cells}

1 or $1.5 \mathrm{ml}$ of cryo solution was added to the cell sediment from the centrifuge. This solution contained $90 \%$ pure FBS and 10\% DMSO. DMSO is a strong solvent in the process of freezing the cells and acts as a protective, preventing the formation of ice crystals and damage to the cell. Then, cryo tubes placed for 2 hours at $-20{ }^{\circ} \mathrm{C}$ temperature. Then, they were placed at $-70^{\circ} \mathrm{C}$ for 24 hours. Next, samples available to transition the nitrogen tank.

\section{Melting down the Freezing cells}

Frozen cells of cryo tube were transferred from liquid nitrogen tank to bain-marie at $37^{\circ} \mathrm{C}$. Then, it was centrifuged by $7 \mathrm{~min}$ and at speed of $1000 \mathrm{rpm}$. Cell sedimentation serum-free medium was added again and centrifuged at $1000 \mathrm{rpm}$ for $7 \mathrm{~min}$ (the same as before). The repetition of this procedure led to complete disappearance of the effect of DMSO. Finally, the cells were transferred to cell culture flasks with fresh medium containing $20-10 \%$ FBS.

\section{MTT assay}

In this study, MDA-MB231 cell line was examined which is one of breast cancer cell lines. Previously, cancer researchers were unable to obtain cell lines of the breast that can survive more than a few weeks [10]. MTT is a laboratory and quantitative test for measurement of cell proliferation in response to various factors and determination of the toxicity of these agents on cells. This method is based on the ability of living cells reductase enzyme in emanating yellow tetrazolium rings MTT to the insoluble crystals and purple formazan which are not able to cross the cell membrane. Then, the crystals dissolved in the isopropanol. During MTT assay, a reaction occurs in living cells as shown in Figure 1.

\section{Results}

Evaluating the trend of Halobacterium salynarum growth

After several bacterial cultures of Halobacterium salynarum and determining the amount of absorption, the standard logarithmically growing curve was drawn at $600 \mathrm{~nm}$ wavelength (Figure 2). The results suggested that the bacteria on days 7 and 8 had the highest growth rate. After this time, bacterial growth was declining. Accordingly, the best time to harvest the bacteria and lipid extraction was the 8 th day.

\section{The profile of nanoparticles}

In this study, homogeneous formulation of paclitaxel nano-archaeosom and a formulation without paclitaxel nano-archaeosom, sonicator, and the homogenizer were used. Their size and surface charge were measured with Zeta sizer and then the results were compared (Figures 3 and 4).

Determine the percentage of capsulation and the amount of drug loading

One $\mathrm{ml}$ of archaeosomes solution containing the drug and the control solution were transferred in two separate micro-tubes. The solutions were centrifuged at a speed of $21000 \mathrm{rpm}$ for 15 minutes. The optical density of the concentration of standard solution of paclitaxel

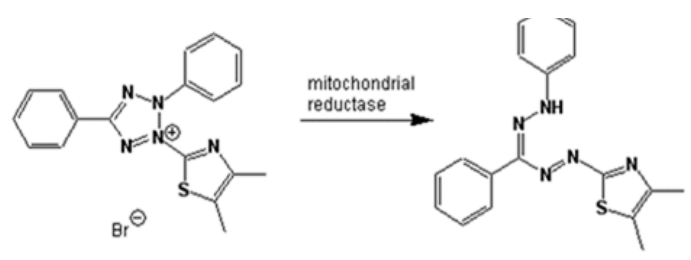

Figure 1. The Reaction That Occurs During the MTT Test in Living Cells 
Table 1. Paclitaxel Absorption in the Wavelength $227 \mathrm{~nm}$

\begin{tabular}{lc}
\hline Absorption & Drug concentration \\
\hline 0 & 0 \\
0.099 & 0.00004875 \\
0.116 & 0.0000975 \\
0.112 & 0.000195 \\
0.125 & 0.00039 \\
0.146 & 0.00078 \\
0.168 & 0.00157 \\
0.259 & 0.00312 \\
0.446 & 0.00625 \\
0.818 & 0.0125 \\
\hline
\end{tabular}

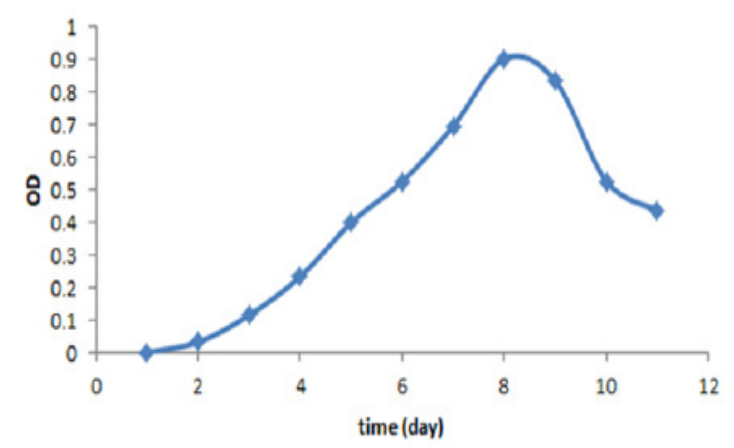

Figure 2. Logarithmic Growth of Halobacterium Salynarum

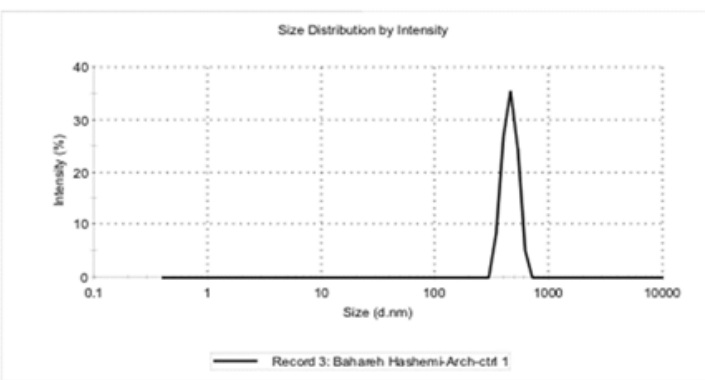

$\overline{\text { Figure 3. Size Distribution Curve Nano-Archaeosom }}$ without Paclitaxel

was determined (Table1). At a wavelength of $227 \mathrm{~nm}$. According to this data, standard chart of paclitaxel and curve slope of the equation were determined for further analysis.

\section{Determination of paclitaxel encapsulation efficiency}

The amount of OD Obtained by the below equation: $Y=14 / 39 x+0 / 091$

OD value of supernatant was 795.0

\section{Discussion}

\section{MTT color measurement}

The results of MTT color measurement using light absorption at a wavelength of nm570 with Elisa reader and
Table 2. Average Results $\mathrm{IC}_{50}$ on Cell Line MDA-MB231

\begin{tabular}{lcc}
\hline Drug formulation $(\mu \mathrm{M})$ & Free drug $(\mu \mathrm{M})$ & The sample \\
\hline 33.7 & 82.9 & $\mathrm{IC}_{50}$ \\
\hline
\end{tabular}

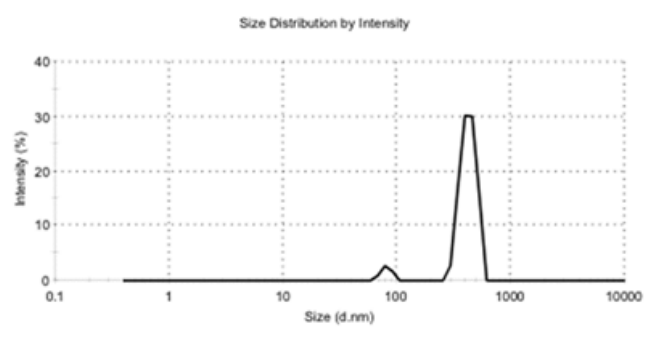

- Recores 5: Bahareh Hestreminver-test 1

$\overline{\text { Figure 4. Size Distribution Curve Nano-Archaeosom }}$ with Paclitaxel

based on the concentration of pharmaceutical formulation used in comparison with amount of cell proliferation was charted. Life of incubation cells with two types of drugs was calculated and compared with the control cells. Results were expressed as the percentage of viable treated cells in the comparison with untreated cells. Survival percentage of breast cancer cells that were loaded with paclitaxel and nano-archaeosom were calculated. The results showed that nano-archaeosomal drug had less $\mathrm{IC}_{50}$ than the free paclitaxel (Table2). Therefore, archaeosomes drug-free formulation had significantly less toxicity on MDA-MB231 cells. The aim of this research was to preparinge a nano-archaeosome formula containing paclitaxel and study the parameters such as encapsulation, particle size, and size distribution. Nano-archaeosomes containing paclitaxel had less inoffensive damage and cytotoxic effect on MDA-MB231 cell line of human breast cancer in comparison with free drug.

As a conclusion, with regard to the benefits of nanocarriers for drug delivery applications due to their small size can penetrate the cells through tiny capillaries. Efficient accumulation of the drug in the purpose sites also causes stable and uniform distribution of the drug for a period of several days or weeks. In this study, the cytotoxicity of paclitaxel in the form of free drug was compared with that of archaeosomal drug in laboratory conditions. To determine the size of archaeosom, Zetasizer device (DLS) was used. The average size of nano- archaeosom particles containing paclitaxel was 430 $\mathrm{nm}$ and its surface charge was -15 whereas the average size of controled nano- archaeosom particles without paclitaxel was $460 \mathrm{~nm}$ and its surface charge was -14 . Zeta potential is a good indication, revealing the amount of repulsive interactions between colloidal particles which is closely linked with the stability of colloidal systems. Under the tested conditions, zeta potential formulations were negative in both cases. Previous studies have shown that negatively charged archaeosom has high physical stability, small particle Previous studies have shown that negatively charged archaeosom has high physical 
stability, small particle size, and high efficacy in trapping of the drug as compared to the positive charge. Similary, nano-archaeosomes containing paclitaxel had higher physical stability than controled nano-archaeosomes in the current study. The results of a study on the amount of drug loading by using Super Nantes separated from the solution showed that the amount of encapsulation of archaeosome containing drug was very convenient. In this study, the effect of archaeosome drug containing paclitaxel was compared with that of archaeosome with drug-free formulation by MTT assay. The findings suggested that archaeosome with drug-free formulation had significantly less toxicity on MDA-MB231 cell line. In addition, we found nano- archaeosomal drug had less $\mathrm{IC}_{50}$ than free paclitax.

\section{Conflict of interest}

The authors declared no conflict of interest.

\section{References}

1.Smith AM, Dave S, Nie S, True L, Gao X, . Multicolor quantum dots for molecular diagnostics of cancer. Expert Rev Mol Diagn. 2006; 6: 231-244.

2. Lacroix, M, Leclercq, G,. Relevance of breast cancer cell lines as models for breast tumours: an update. Breast Cancer Research and Treatment. 2004; 83, 249-289.

3. Horwitz SB,. Mechanism of action of taxol. Trends Pharmacol Sci. 1992; 13:134-136.

4. Francesco Fabbri; et al,. Sequential events of apoptosis involving docetaxel, a microtubule-interfering agent: A cytometric study. BMC Cell Bio, 2006; 5: 7:6.

5. Sweetman, SC, editor, Martindale, the complete drug reference. London: pharmaceutical press. 2002; 16:534-536.

6. Crook St, Prestaydo A, eds,. Cancer and Chemotherapy: introduction to neoplasia and antineoplastic chemotherapy. New York: Academic Press. 1980; 12:77-92.

7. M.L. Fjallskog, L. Frii, J. Bergh, Is cremophor,. Solvent for paclitaxel, cytotoxic. Lancet. 1993; 5: 342-876.

8. A. Sharma, S.V. Balasubramanian, R.M. Straubinger,. Pharmaceutical and physical properties of paclitaxel complexes with cyclodextrins. Am. Chem. Soc. Am. Pharm. Ass. 1995; 84, 1223-1230.

9. Key T., Verkasalo P., and Banks E,. Epidemiology of breast cancer. Lancet Oncol. 2001; 2: 133-140.

10. Armstrong K, Eisen A, Weber B,. Assessing the risk of breast cancer. N Engl J Med. 2000; 342 (8):564-71.

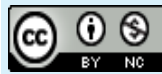

This work is licensed under a Creative Commons AttributionNon Commercial 4.0 International License. 